\title{
Reduced Order Projective and Hybrid Projective Combination-Combination Synchronization of Four Chaotic Josephson Junctions
}

\author{
K. S. Ojo, ${ }^{1,2}$ A. N. Njah, ${ }^{2}$ O. I. Olusola, ${ }^{1}$ and M. O. Omeike ${ }^{3}$ \\ ${ }^{1}$ Department of Physics, University of Agriculture, Abeokuta, Nigeria \\ ${ }^{2}$ Department of Physics, University of Lagos, Lagos, Nigeria \\ ${ }^{3}$ Department of Mathematics, University of Agriculture, Abeokuta, Nigeria \\ Correspondence should be addressed to K. S. Ojo; kaystephe@yahoo.com
}

Received 10 October 2013; Revised 23 December 2013; Accepted 1 January 2014; Published 13 February 2014

Academic Editor: Min W. Lee

Copyright (C) 2014 K. S. Ojo et al. This is an open access article distributed under the Creative Commons Attribution License, which permits unrestricted use, distribution, and reproduction in any medium, provided the original work is properly cited.

\begin{abstract}
This paper investigates the reduced order projective and hybrid projective combination-combination synchronization of four chaotic Josephson junctions consisting of two third order Josephson junctions as the drives and two second order chaotic Josephson junctions as the response systems via active backstepping technique. The investigation confirms the achievement of reduced order projective and hybrid projective combination-combination synchronization among four chaotic Josephson junctions via active backstepping technique. Numerical simulations are validated to show the effectiveness of the synchronization scheme. Reduced order combination-combination synchronization scheme has more significant applications to neural encoding and decoding of information in biological systems and to the security of information transmission in communication systems than the usual one drive system and one response system synchronization scheme.
\end{abstract}

\section{Introduction}

One of the most significant phenomenon in nonlinear science is that of synchronization of dynamical systems. Synchronization classically represents the entrainment of frequencies of oscillations due to weak interactions between two or more dynamical systems. Many synchronization types and schemes have been discovered and reported, such as complete synchronization [1], phase synchronization [2], generalized synchronization [3], lag synchronization [4], antisynchronization [5], hybrid synchronization [6], projective synchronization [7], projective hybrid synchronization [8], function projective synchronization [9], increased order synchronization [10], and reduced order synchronization [11]. The discovery of various types of synchronization is due to the prospective applications of synchronization in especially chemical reactions, power converter, biological systems, information processing, and secure communications. In search of effective and efficient methods of synchronization of chaotic systems, various linear and nonlinear control techniques have been developed which include slide mode control [12], adaptive control [13], active control [14,15], impulsive control [16], linear feedback control [17], and backstepping control [18-21]. Meanwhile, the active control and backstepping techniques have been reported as efficient and excellent nonlinear control method for synchronization of either identical or nonidentical chaotic systems [21, 22]. Based on the above discussion, a more powerful method which is the combination of active control and backstepping techniques known as active backstepping technique is chosen as our preferred method of synchronization.

Projective synchronization behaviour was first reported by Mainieri and Rehacek [23], where they explained the mechanism of the realization of projective synchronization 
in three-dimensional partially linear systems. Projective synchronization refers to the dynamical behaviour in which the responses of two or more systems synchronize up to a constant scaling factor $\alpha \in \mathfrak{R}$ [24], where complete synchronization and antisynchronization can be regarded as the special cases of projective synchronization with $\alpha=$ 1 and $\beta=-1$, respectively. Projective synchronization has been extensively studied because faster communication can be realized with its proportional feature [6]. Hybrid synchronization scheme is a synchronization scheme where one part of the system is antisynchronized and the other part is completely synchronized such that complete synchronization and antisynchronization coexist in the system [6]. The coexistence of complete synchronization and antisynchronization enhances security in communication and chaotic encryption schemes. Hybrid projective synchronization is a synchronization scheme where one part of the system synchronizes up to a constant positive scaling factor $\alpha \neq 1$, while the other part of the system synchronizes up to a constant negative scaling factor $\beta \neq-1$, so projective hybrid synchronization is a combination of hybrid and projective synchronization.

Synchronization of chaos in nonlinear dynamical systems with different dynamical structures and orders, called reduced order or increased order synchronization, has not been adequately explored despite its occurrence in biological science and social sciences [25-27]. For instance, synchronous activity is inevitable in the thalamic and hippocampal neurons network though the network consists of neurons of different dynamical structures and orders [26]. Furthermore, synchronization between the circulatory system and respiratory system may involve dynamical organs with different structures and orders [11]. In fact, synchronization of systems of different dynamical structures and orders can also be observed between the heart and the lung [28]. The main characteristic of reduced order synchronization is that the order of the master is higher than that of the slave and all the state variables of the slave system are synchronized with those of the master. The synchronization of dynamical systems with different structures and orders or reduced order synchronization is very challenging and very important from the perspective of real life applications.

Most of the studies on synchronization of chaotic systems have been restricted to one drive system and one response system which has limited flexibility and applicability to real world systems such as insecure communication. However, recently, few scientific researches were published on synchronization of chaotic systems of the same order consisting of three chaotic systems and four chaotic systems which are called combination synchronization and combination-combination synchronization, respectively [29-31]. The previous research works on combination synchronization and combination-combination synchronization mainly focus their numerical simulation results on complete synchronization and not on projective synchronization or hybrid projective synchronization [29-31]. Combination synchronization of chaotic systems of different orders has been considered [32], while a more challenging case of combination-combination synchronization of systems of different orders is not yet investigated. Also, Josephson junction has not been used as a model for investigating combination or combination-combination synchronization despite its great physical importance. The reduced order combination-combination proposed in this paper is more generalized such that reduced order combination synchronization is just a specific case of this proposed scheme. This paper also provides numerical results of evidence of projective and hybrid projective synchronization which has not been considered in previous papers on combinationcombination synchronization.

The projective and hybrid projective combinationcombination synchronization scheme presented in this paper has more flexibility and applicability to real life system; for example, in secure communication scheme information signal can be divided into several parts; then each part is loaded in each of the different drive systems and after synchronization the original information signal is retrieved by combining the received signals from different response systems correctly [31]. The case for the projective combination-combination synchronization can be used to achieve fast communication due to the proportionality between the synchronized dynamical states, while the case for projective hybrid synchronization can offer the opportunity of transforming digital signals through the continuous transformation between synchronization and antisynchronization which will further enhance security in communication and chaotic encryption schemes [33]. Moreover, combinationcombination synchronization of different orders gives better insight into the synchronization of biological systems wherein synchronization of different organs of different dynamical structures and orders is involved. Reduced order combination-combination synchronization gives a better understanding of synchronization phenomenon in complex biological systems since synchronization in real life system is complex. The aim of this work is to present projective and hybrid projective reduced order combination-combination synchronization of four chaotic Josephson junctions. This problem of projective and hybrid projective reduced order combination-combination synchronization is reported here for the first time to the best of our knowledge.

The rest of this paper is organized as follows. Section 2 gives the description of the systems. Section 3 presents reduced order combination-combination synchronization scheme of four chaotic systems. Section 4 deals with generalized reduced order combination-combination synchronization between two third order Josephson junctions as the drive systems and two second order Josephson junction as the response systems via active backstepping technique with projective and hybrid projective combination-combination synchronization as a special cases, while Section 5 concludes the paper.

\section{Description of Josephson Junctions}

2.1. Resistive-Capacitive-Inductive Shunted Josephson Junction (RCISJJ). The resistive-capacitive-inductive shunted 
Josephson junction in dimensionless form is described by the set of first order differential equations below:

$$
\begin{gathered}
\dot{x}=y, \\
\dot{y}=\frac{1}{\beta_{C}}(i-g(y) y-\sin x-z), \\
\dot{z}=\frac{1}{\beta_{L}}(y-z),
\end{gathered}
$$

where $g(y)$ is the nonlinear damping function approximated by current-voltage relation between the junctions and is defined by

$$
g(y)= \begin{cases}0.366 & \text { if }|y|>2.9 \\ 0.061 & \text { if }|y| \leq 2.9\end{cases}
$$

$x, y$, and $z$ represent the phase difference, the voltage in the junction, and the inductive current, respectively. $\beta_{C}$ and $\beta_{L}$ are capacitive and inductive constant, respectively. $i$ is the external direct current. Figure 1 shows the chaotic attractor of the RCI shunted Josephson junction for the following set of parameters: $i=1<i<1.3, \beta_{C}=2.6$, and $\beta_{L}=$ 0.707 , with the initial conditions $(x, y, z)=(0,0,0)$. The RCI shunted Josephson has been found to be more appropriate in high frequency applications. In RCI Josephson junction chaotic oscillation has been modulated in response to both the amplitude and frequency of an external sinusoidal signal.

\subsection{Resistive-Capacitive Shunted Josephson Junction (RCSJJ).} The resistive-capacitive Josephson junction under the external periodic force is given by the second order differential equation below:

$$
\ddot{\phi}=-\alpha \dot{\phi}-\sin \phi+a+b \sin \omega t,
$$

where $\phi$ is the phase difference between quantum mechanical wave functions of two superconductor junctions separated by some nonsuperconducting material or barrier. $\alpha$ and $a$ are the dimensionless damping and applied current. $b \sin \omega t$ is the external periodic sinusoidal force. $b$ and $\omega$, respectively, are the amplitude and frequency of the external periodic sinusoidal force. The second order differential equation in (3) can be transformed into a set of first order differential equations as follows:

$$
\begin{gathered}
\dot{x}=y, \\
\dot{y}=-\alpha y-\sin x+a+b \sin \omega t .
\end{gathered}
$$

Figure 2 shows the chaotic attractor for resistive-capacitive shunted Josephson junction using the following parameter values: $\alpha=0.5, a=0.89, b=0.4$, and $\omega=0.25$.

\section{Reduced Order Combination-Combination Synchronization Scheme}

In this drive response scheme, combination synchronization of four chaotic systems will be considered. Suppose we have

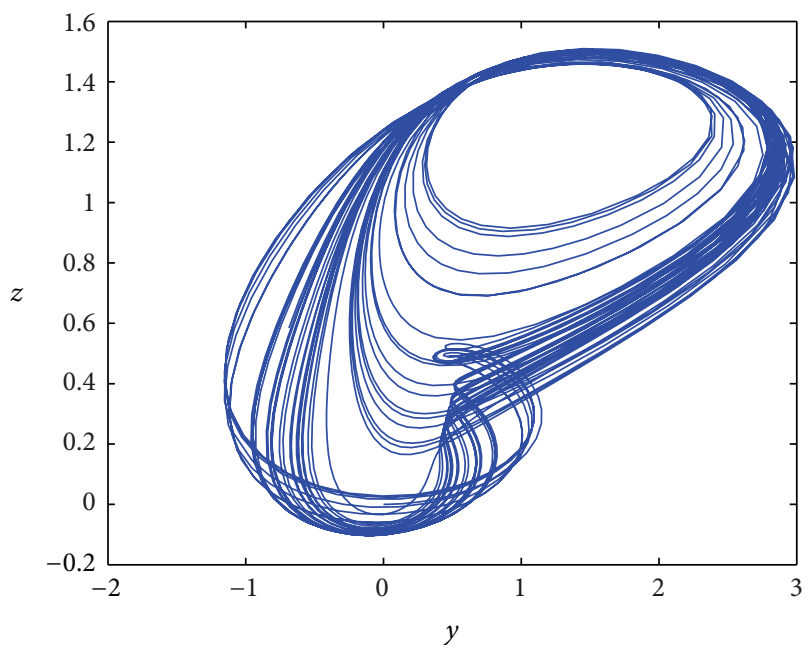

FIGURE 1: Phase portrait of chaotic attractors of resistive-capacitiveinductive Josephson junction.

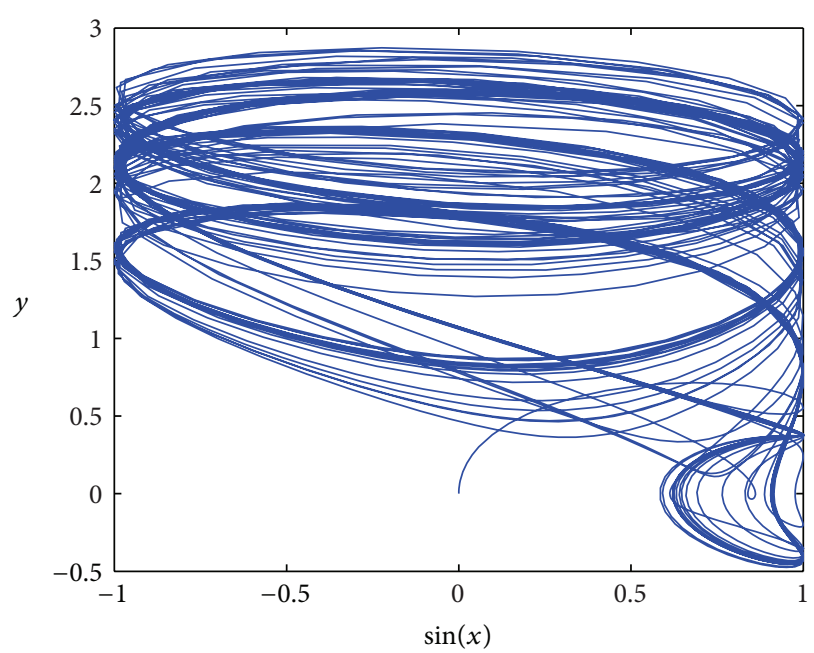

FIGURE 2: Phase portrait of chaotic attractors of resistive-capacitive shunted Josephson junction.

two drive systems and two response systems. The first drive system is given as

$$
\dot{x}=A x+f(x),
$$

where $x=\left(x_{1}, x_{2}, \ldots, x_{n}\right)^{T} \in \mathfrak{R}^{n}, A \in \mathfrak{R}^{n} \times \mathfrak{R}^{n}, A$ is a constant matrix, $n$ is the order of the system, and $f(x)$ is nonlinear function of the system which is continuous and differentiable. The second drive system is given as

$$
\dot{y}=B y+f(y)
$$

where $y=\left(y_{1}, y_{2}, \ldots, y_{m}\right)^{T} \in \mathfrak{R}^{m}, B \in \mathfrak{R}^{m} \times \mathfrak{R}^{m}, B$ is a constant matrix, $m$ is the order of the system, and $f(y)$ is 
nonlinear function of the system which is continuous and differentiable. The first response system is given as

$$
\dot{z}=C z+f(z)+u(x, y, z, w)
$$

where $z=\left(z_{1}, z_{2}, \ldots, z_{q}\right)^{T} \in \mathfrak{R}^{q}, C \in \mathfrak{R}^{q} \times \mathfrak{R}^{q}$ is a constant matrix, $q$ is the order of the matrix, and $f(z)$ is nonlinear function of the system which is continuous and differentiable. The second response system is given as

$$
\dot{w}=D w+f(w)+v(x, y, z, w),
$$

where $w=\left(w_{1}, w_{2}, \ldots, w_{p}\right)^{T} \in \mathfrak{R}^{p}, D \in \mathfrak{R}^{p} \times$ $\mathfrak{R}^{p}$ is a constant system matrix, $p$ is the order of the matrix $D, f(w)$ is nonlinear function of the system which is continuous and differentiable, and $v(x, y, z, w)=$ $\left(v_{1}(x, y, z, w), v_{2}(x, y, z, w), \ldots, w_{p}(x, y, z, w)\right)^{T} \in \mathfrak{R}^{p}$ are the controllers to be designed.

Definition 1. The drive systems (5) and (4) and the response systems (7) and (8) are said to achieve generalized reduced order combination-combination synchronization if there exist four constant matrices $M_{1} \in \mathfrak{R}^{n}, M_{2} \in \mathfrak{R}^{m}, M_{3} \in \mathfrak{R}^{q}$, and $M_{4} \in \mathfrak{R}^{p}(m, n>q, p)$ such that $\lim _{t \rightarrow \infty} \|\left(M_{4} w+M_{3} z\right)-$ $\left(M_{1} x+M_{2} y\right) \|=0$, where $\|\cdot\|$ represents the matrix norm.

Remark 2. The constant matrices $M_{1}, M_{2}, M_{3}$, and $M_{4}$ are called scaling matrices.

Remark 3. The generalized reduced order combination synchronization scheme above can lead to reduced order modified projective synchronization, reduced order projective synchronization, reduced order antisynchronization, and asymptotic stability depending on the values assigned to the scaling matrices $M_{1}, M_{2}, M_{3}$, and $M_{4}$.

\section{Reduced Order Combination-Combination Synchronization of Two Third Order and Two Second Order Josephson Junctions}

4.1. Design of Controller via Active Backstepping Technique. In this section, two third order Josephson junctions in (9) and (10) are taken as the drive systems while the two second order nonautonomous Josephson junctions in (11) and (12) are taken as the response systems in order to achieve generalized reduced order combination-combination synchronization among the four chaotic Josephson junctions as follows:

$$
\begin{gathered}
\dot{x}_{1}=x_{2}, \\
\dot{x}_{2}=\frac{1}{\beta_{C}}\left(i-g\left(x_{2}\right) x_{2}-\sin x_{1}-x_{3}\right), \\
\dot{x}_{3}=\frac{1}{\beta_{L}}\left(x_{2}-x_{3}\right) .
\end{gathered}
$$

The second drive system is

$$
\begin{gathered}
\dot{y}_{1}=y_{2}, \\
\dot{y}_{2}=\frac{1}{\beta_{C}}\left(i-g\left(y_{2}\right) y_{2}-\sin y_{1}-y_{3}\right), \\
\dot{y}_{3}=\frac{1}{\beta_{L}}\left(y_{2}-y_{3}\right) .
\end{gathered}
$$

The first response system is given as

$$
\begin{gathered}
\dot{z}_{1}=z_{2}+u_{1}, \\
\dot{z}_{2}=-\alpha z_{2}-\sin z_{1}+a+b \sin \omega t+u_{2} .
\end{gathered}
$$

The second response system is given as

$$
\begin{gathered}
\dot{w}_{1}=w_{2}+u_{3}, \\
\dot{w}_{2}=-\alpha w_{2}-\sin w_{1}+a+b \sin \omega t+u_{4},
\end{gathered}
$$

where $u_{1}, u_{2}, u_{3}$, and $u_{4}$ are the controllers to be designed. We define the error systems as follows:

$$
\begin{gathered}
e_{1}=\delta_{1} w_{1}+\gamma_{1} z_{1}-\left(\alpha_{1} x_{1}+\beta_{1} y_{1}+\alpha_{3} x_{3}+\beta_{3} y_{3}\right) \\
e_{2}=\delta_{2} w_{2}+\gamma_{2} z_{2}-\left(\alpha_{2} x_{2}+\beta_{2} y_{2}\right)
\end{gathered}
$$

Using the error systems (13) and the systems defined in (9)(12) yields the following error dynamics:

$$
\begin{aligned}
\dot{e}_{1}= & \delta_{1} w_{2}+\gamma_{1} z_{2}-\alpha_{1} x_{2}-\beta_{1} y_{2}-\frac{\alpha_{3}}{\beta_{L}}\left(x_{2}-x_{3}\right) \\
& -\frac{\beta_{3}}{\beta_{L}}\left(y_{2}-y_{3}\right)+\delta_{1} u_{3}+\gamma_{1} u_{1} \\
= & \frac{\delta_{1}}{\delta_{2}}\left(e_{2}+\alpha_{2} x_{2}+\beta_{2} y_{2}-\gamma_{2} z_{2}\right)-\alpha_{1} x_{2} \\
& -\beta_{1} y_{2}-\frac{\alpha_{3}}{\beta_{L}}\left(x_{2}-x_{3}\right)-\frac{\beta_{3}}{\beta_{L}}\left(y_{2}-y_{3}\right) \\
& +\gamma_{1} z_{2}+\delta_{1} u_{3}+\gamma_{1} u_{1}, \\
\dot{e}_{2}= & \delta_{2}\left(-\alpha w_{2}-\sin w_{1}+a+b \sin \omega t+u_{4}\right) \\
& +\gamma_{2}\left(-\alpha z_{2}-\sin z_{1}+a+b \sin \omega t+u_{2}\right) \\
& -\frac{\alpha_{2}}{\beta_{C}}\left(i-g\left(x_{2}\right) x_{2}-\sin x_{1}-x_{3}\right) \\
& -\frac{\beta_{2}}{\beta_{C}}\left(i-g\left(y_{2}\right) y_{2}-\sin y_{1}-y_{3}\right) \\
= & -\alpha\left(e_{2}-\gamma_{2} z_{2}+\alpha_{2} x_{2}+\beta_{2} y_{2}\right) \\
& +\delta_{2}\left(-\sin w_{1}+a+b \sin \omega t\right) \\
& +\gamma_{2}\left(\alpha z_{2}-\sin z_{1}+a+b \sin \omega t\right)
\end{aligned}
$$




$$
\begin{aligned}
& -\frac{\alpha_{2}}{\beta_{C}}\left(i-g\left(x_{2}\right) x_{2}-\sin x_{1}-x_{3}\right) \\
& -\frac{\beta_{2}}{\beta_{C}}\left(i-g\left(y_{2}\right) y_{2}-\sin y_{1}-y_{3}\right) \\
& +\delta_{2} u_{4}+\gamma_{2} u_{2} .
\end{aligned}
$$

Thus, the error dynamics of the system can be written as

$$
\begin{aligned}
& \dot{e}_{1}=\frac{\delta_{1}}{\delta_{2}} e_{2}+A_{1}+U_{1}, \\
& \dot{e}_{2}=-\alpha e_{2}+A_{2}+U_{2},
\end{aligned}
$$

where

$$
\begin{gathered}
A_{1}=\frac{\delta_{1}}{\delta_{2}}\left(\alpha_{2} x_{2}+\beta_{2} y_{2}-\gamma_{2} z_{2}\right)-\alpha_{1} x_{2}-\beta_{1} y_{2} \\
+\gamma_{1} z_{2}-\frac{\alpha_{3}}{\beta_{L}}\left(x_{2}-x_{3}\right)-\frac{\beta_{3}}{\beta_{L}}\left(y_{2}-y_{3}\right), \\
A_{2}=-\alpha\left(\alpha_{2} x_{2}+\beta_{2} y_{2}\right)+\gamma_{2}\left(-\sin z_{1}+a+b \sin \omega t\right) \\
+\delta_{2}\left(-\sin w_{1}+a+b \sin \omega t\right) \\
-\frac{\alpha_{2}}{\beta_{C}}\left(i-g\left(x_{2}\right) x_{2}-\sin x_{1}-x_{3}\right) \\
-\frac{\beta_{2}}{\beta_{C}}\left(i-g\left(y_{2}\right) y_{2}-\sin y_{1}-y_{3}\right), \\
U_{1}=\gamma_{1} u_{1}+\delta_{1} u_{3}, \\
U_{2}=\gamma_{2} u_{2}+\delta_{2} u_{4} .
\end{gathered}
$$

Then, we obtain the following results.

Theorem 4. If the controllers are chosen as

$$
\begin{aligned}
U_{1}= & \frac{\alpha_{3}}{\beta_{L}}\left(x_{2}-x_{3}\right)+\frac{\beta_{3}}{\beta_{L}}\left(y_{2}-y_{3}\right)-\gamma_{1} z_{2}-k q_{1} \\
& -\frac{\delta_{1}}{\delta_{2}}\left(\alpha_{2} x_{2}+\beta_{2} y_{2}+\gamma_{2} z_{2}\right)+\alpha_{1} x_{2}+\beta_{1} y_{2}, \\
U_{2}= & \alpha\left(\alpha_{2} x_{2}+\beta_{2} y_{2}\right)-\gamma_{2}\left(-\sin z_{1}+a+b \sin \omega t\right) \\
& -\delta_{2}\left(-\sin w_{1}+a+b \sin \omega t\right)-k q_{2} \\
& +\frac{\alpha_{2}}{\beta_{C}}\left(i-g\left(x_{2}\right) x_{2}-\sin x_{1}-x_{3}\right) \\
& +\frac{\beta_{2}}{\beta_{C}}\left(i-g\left(y_{2}\right) y_{2}-\sin y_{1}-y_{3}\right)+\alpha q_{2}-\frac{\delta_{1}}{\delta_{2}} q_{1},
\end{aligned}
$$

where $q_{1}=e_{1}$ and $q_{2}=e_{2}$, then, the drive systems (9) and (10) will achieve reduced order combination-combination synchronization with the response systems (11) and (12).
Proof. Our goal is to find the control functions which will enable the drive systems (9) and (10) to achieve reduced combination-combination synchronization with the response systems (11) and (12) via the active backstepping technique. The design procedures include three steps as shown below.

Step 1. Let $q_{1}=e_{1}$, its time derivative is

$$
\dot{q}_{1}=\dot{e}_{1}=\frac{\delta_{1}}{\delta_{2}} e_{2}+U_{1}+A_{1},
$$

where $e_{2}=\alpha_{1}\left(q_{1}\right)$ can be regarded as virtual controller. In order to stabilize $q_{1}$-subsystem, we choose the Lyapunov function $v_{1}=(1 / 2) q_{1}^{2}$. The time derivative of $v_{1}$ is

$$
\dot{v}_{1}=q_{1} \dot{q}_{1}=q_{1}\left(\frac{\delta_{1}}{\delta_{2}} \alpha_{1}\left(q_{1}\right)+A_{1}+U_{1}\right) .
$$

Suppose $\alpha_{1}\left(q_{1}\right)=0$ and the control function $U_{1}$ is chosen as

$$
U_{1}=-\left(A_{1}+k q_{1}\right) ;
$$

then $\dot{v}_{1}=-k q_{1}^{2}<0$, where $k$ is a positive constant which represents the feedback gain. Then, $\dot{v}_{1}$ is negative definite and the subsystem $q_{1}$ is asymptotically stable. Since the virtual controller $\alpha_{1}\left(q_{1}\right)$ is estimative, the error between $e_{2}$ and $\alpha_{1}\left(q_{1}\right)$ can be denoted by $q_{2}=e_{2}-\alpha_{1}\left(q_{1}\right)$. Thus, we have the following $\left(q_{1}, q_{2}\right)$ subsystems:

$$
\begin{gathered}
\dot{q}_{1}=\frac{\delta_{1}}{\delta_{2}} q_{2}-k q_{1}, \\
\dot{q}_{2}=-\alpha q_{2}+U_{2}+A_{2} .
\end{gathered}
$$

Step 2. In order to stabilize subsystem (21), the following Lyapunov function can be chosen as $v_{2}=v_{1}+(1 / 2) q_{2}^{2}$. Its time derivative is

$$
\dot{v}_{2}=-k q_{1}^{2}+q_{2}\left(\frac{\delta_{1}}{\delta_{2}} q_{1}-\alpha q_{2}+U_{2}+A_{2}\right) .
$$

If the control function $U_{2}$ is chosen as

$$
U_{2}=-A_{2}-k q_{2}+\alpha q_{2}-\frac{\delta_{1}}{\delta_{2}} q_{1},
$$

then $\dot{v}_{2}=-k q_{1}^{2}-k q_{2}^{2}<0$, where $k$ is a positive constant which represents the feedback gain. Then, $\dot{v}_{2}$ is negative definite and the subsystems $\left(q_{1}, q_{2}\right)$ in (21) are asymptotically stable. This implies that reduced order combination-combination synchronization of the drive systems (9) and (10) with the response systems (11) and (12) is achieved. Finally, the subsystem (21) becomes

$$
\begin{gathered}
\dot{q}_{1}=\frac{\delta_{1}}{\delta_{2}} q_{2}-k q_{1}, \\
\dot{q}_{2}=-\frac{\delta_{1}}{\delta_{2}} q_{1}-k q_{2} .
\end{gathered}
$$

This completes the proof. Several corollaries can be deduced from Theorem 4. However, we will consider only one corollary related to our result. 


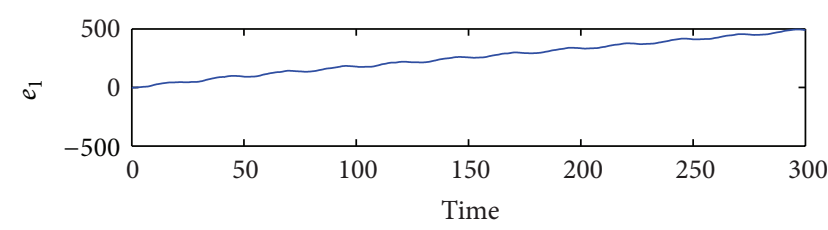

(a)

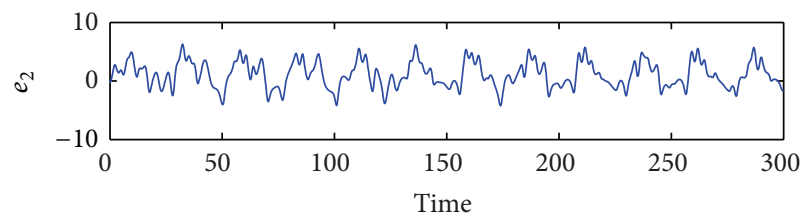

(b)

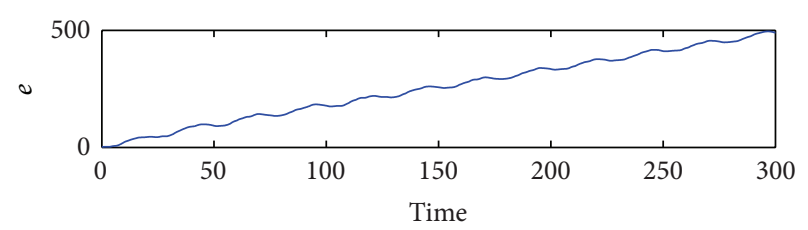

(c)

FIGURE 3: Error dynamics of the drive and the response systems with controllers deactivated for $0 \leq t \leq 300$, where $e_{1}=w_{1}+z_{1}-\left(0.5 x_{1}+\right.$ $\left.0.7 y_{1}+0.2 x_{3}+0.3 y_{3}\right), e_{2}=w_{2}+z_{2}-\left(0.4 x_{2}+1.2 y_{2}\right)$, and $e=\sqrt{e_{1}^{2}+e_{2}^{2}}$.

Suppose $\delta_{1}=\delta_{2}=\gamma_{1}=\gamma_{2}=1, u_{1}=u_{3}$, and $u_{2}=u_{4}$ in (17); then, we have Corollary 5.

Corollary 5. If the controllers are chosen as

$$
\begin{aligned}
u_{1}=u_{3}=\frac{1}{2}( & \left(\alpha_{1}-\alpha_{2}\right) x_{2}+\left(\beta_{1}-\beta_{2}\right) y_{2} \\
& \left.+\frac{\alpha_{3}}{\beta_{L}}\left(x_{2}-x_{3}\right)+\frac{\beta_{3}}{\beta_{L}}\left(y_{2}-y_{3}\right)-k q_{1}\right), \\
u_{2}=u_{4}=\frac{1}{2}( & (\alpha-k) q_{2}-q_{1}+\alpha\left(\alpha_{2} x_{2}+\beta_{2} y_{2}\right) \\
& +\left(\sin z_{1}-a-b \sin \omega t\right) \\
& +\left(\sin w_{1}-a-b \sin \omega t\right) \\
& +\frac{\alpha_{2}}{\beta_{C}}\left(i-g\left(x_{2}\right) x_{2}-\sin x_{1}-x_{3}\right) \\
& \left.+\frac{\beta_{2}}{\beta_{C}}\left(i-g\left(y_{2}\right) y_{2}-\sin y_{1}-y_{3}\right)\right)
\end{aligned}
$$

where $q_{1}=w_{1}+z_{1}-\left(\alpha_{1} x_{1}+\beta_{1} y_{1}+\alpha_{3} x_{3}+\beta_{3} y_{3}\right)$ and $q_{2}=w_{2}+z_{2}-\left(\alpha_{2} x_{2}+\beta_{2} y_{2}\right)$, then, the drive systems (9) and (10) achieve generalized reduced order combination-combination synchronization with the response systems (11) and (12).

Two cases of Corollary 5 will be considered as follows.

Case 1. Suppose the scaling parameters are chosen as $\alpha_{1}=$ $0.5, \alpha_{2}=0.4, \alpha_{3}=0.2, \beta_{1}=0.7, \beta_{2}=1.2$, and $\beta_{3}=0.3$; then,

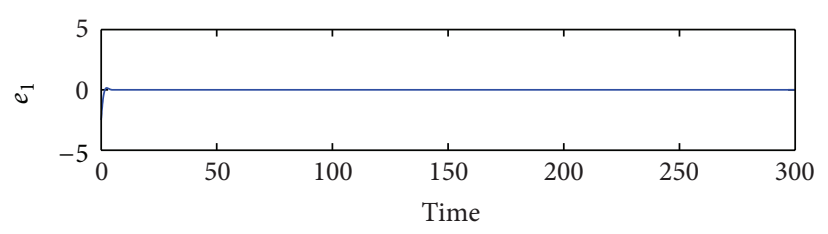

(a)

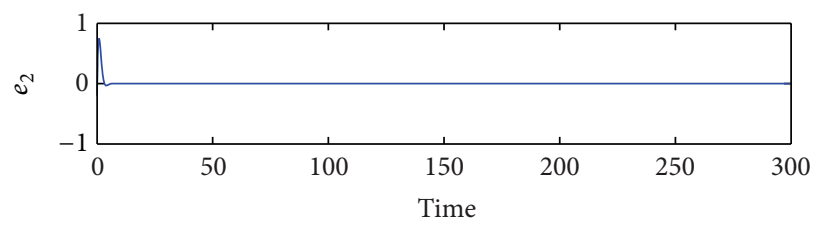

(b)

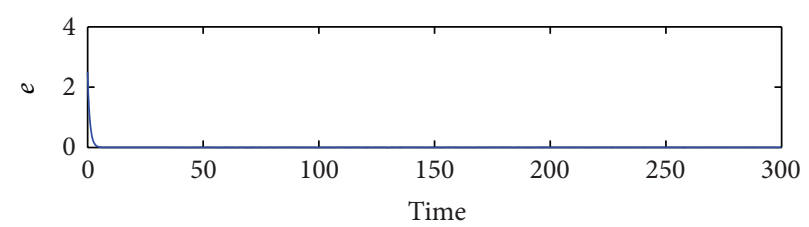

(c)

FIGURE 4: Error dynamics of the drive and the response systems with controllers activated for $t \geq 0$, where $e_{1}=w_{1}+z_{1}-\left(0.5 x_{1}+0.7 y_{1}+\right.$ $\left.0.2 x_{3}+0.3 y_{3}\right), e_{2}=w_{2}+z_{2}-\left(0.4 x_{2}+1.2 y_{2}\right)$, and $e=\sqrt{e_{1}^{2}+e_{2}^{2}}$.

the drive systems (9) and (10) achieve reduced order generalized projective combination-combination synchronization with the response systems (11) and (12).

Case 2. Suppose the scaling factors are chosen as $\alpha_{1}=$ $0.5, \alpha_{2}=-0.4, \alpha_{3}=0.2, \beta_{1}=0.7, \beta_{2}=-1.2$, and $\beta_{3}=0.3$; then, the drive systems (9) and (10) achieve reduced order generalized hybrid projective combinationcombination synchronization with the response systems (11) and (12).

4.2. Numerical Simulation Results. To verify the effectiveness of the designed controllers, we used ode 45 fourth order Runge-Kutta algorithm run on MATLAB. In the numerical simulation procedure, we use the system parameter values as shown in Figures 1 and 2 to ensure chaotic dynamics of the state variables. The initial conditions of the drive systems and response systems are given, respectively, as $\left(x_{1}, x_{2}, x_{3}\right)=(0,0,0),\left(y_{1}, y_{2}, y_{3}\right)=(1,1,1),\left(z_{1}, z_{2}\right)=$ $(2,2)$, and $\left(w_{1}, w_{2}\right)=(2,2)$. The numerical results will be considered under two cases.

Case 3. The numerical results are as follows. Figure 3 shows the error dynamics of the state variables when the controllers are deactivated for $t \geq 0$; Figure 4 shows that reduced order generalized projective combination-combination synchronization is achieved between the two third order drive JJs and the two second order response JJs as indicated by the convergence of the error dynamic of state variables to zero as soon as the controllers are switched on for $t \geq 0$; and Figure 5 shows the projection of the state variables of 


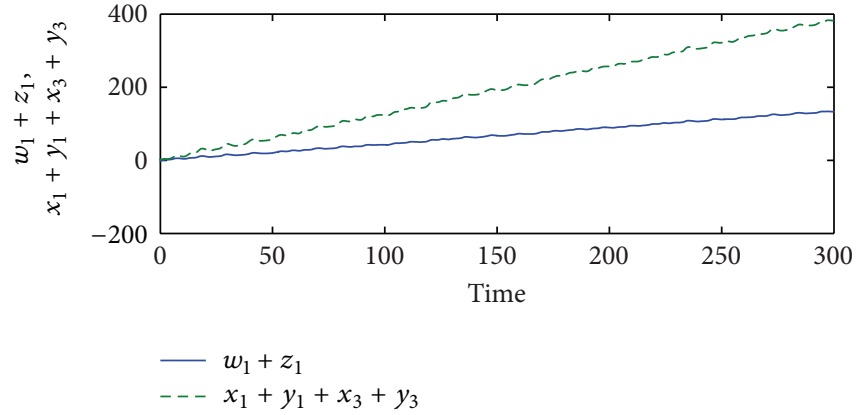

(a)

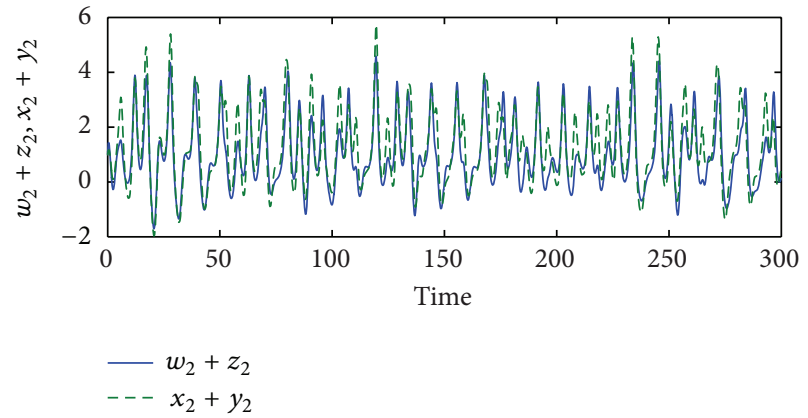

(b)

FIgure 5: Dynamics of the drive (dashed line) and the response (solid line) variables with controllers at $t \geq 0$.

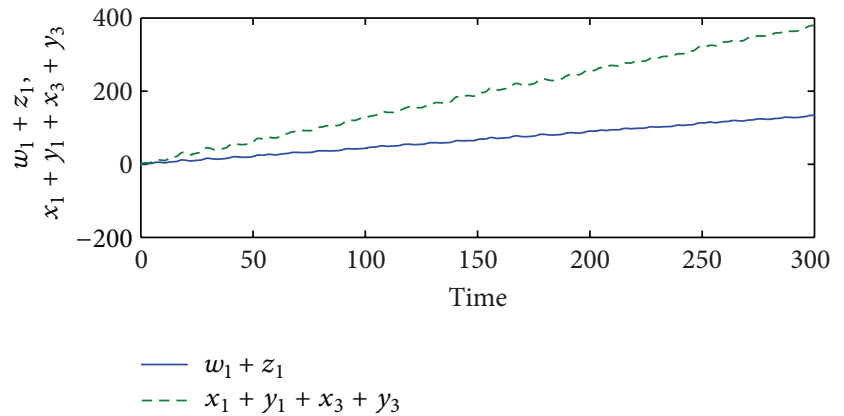

(a)

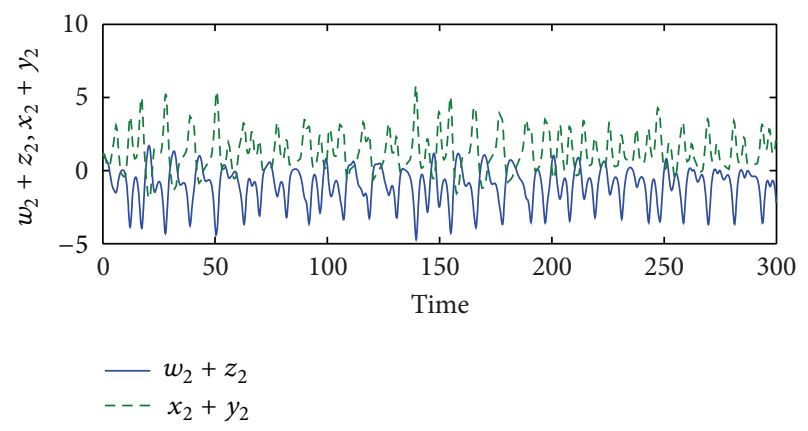

(b)

FIgURE 6: Dynamics of the drives (dashed line) and the response (solid line) variables with controllers activated for $t \geq 0$.

the third order JJs (drive) on the second order JJs (response) when the controllers are activated for $t \geq 0$ which again confirms reduced order generalized projective combinationcombination synchronization among the systems.

Case 4. The numerical results are as follows. Figure 7 shows the error dynamics of the state variables when the controllers are deactivated for $t \geq 0$; Figure 8 shows that reduced order generalized hybrid projective combination-combination synchronization is achieved between the two third order drive JJs and the two second order response JJs as indicated by the convergence of the error dynamic of state variables to zero as soon as the controllers are switched on for $t \geq 0$; and

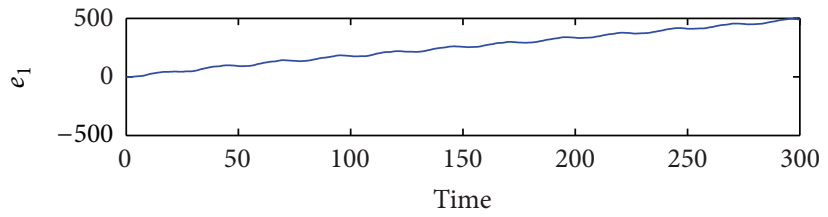

(a)

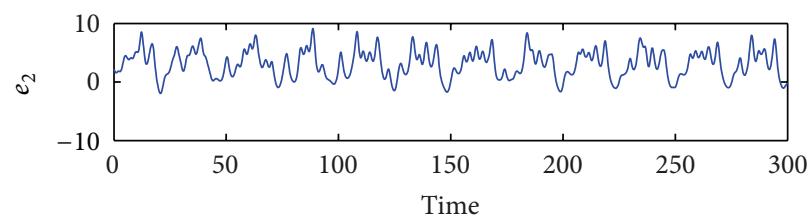

(b)

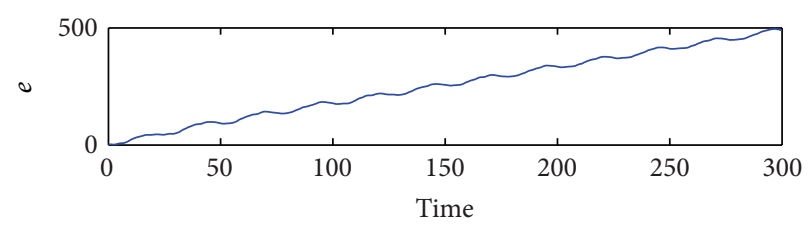

(c)

FIGURE 7: Error dynamics of the drive and the response systems with controllers deactivated for $0 \leq t \geq 300$, where $e_{1}=w_{1}+z_{1}-\left(0.5 x_{1}+\right.$ $\left.0.7 y_{1}+0.2 x_{3}+0.3 y_{3}\right), e_{2}=w_{2}+z_{2}+\left(0.4 x_{2}+1.2 y_{2}\right)$, and $e=\sqrt{e_{1}^{2}+e_{2}^{2}}$.

Figure 6 shows the projection of the state variables of the third order JJs (drive) on the second order JJs (response) when the controllers are activated for $t \geq 0$ which again confirms reduced order generalized hybrid projective combinationcombination synchronization among the systems.

\section{Conclusion}

We employed the active backstepping technique to achieve reduced-order generalized projective and hybrid projective combination-combination synchronization of four chaotic Josephson junctions consisting of two third order chaotic Josephson junctions as drives and two second order chaotic Josephson junctions as response systems. The theoretical analysis revealed that with a suitable choice of controllers 


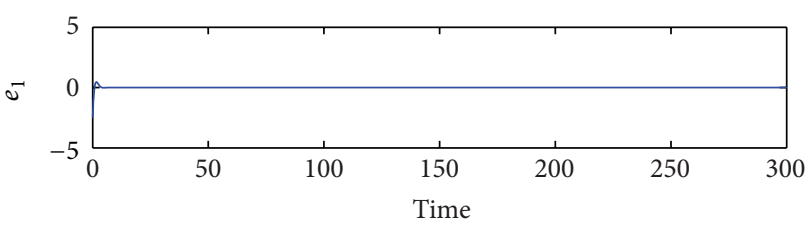

(a)

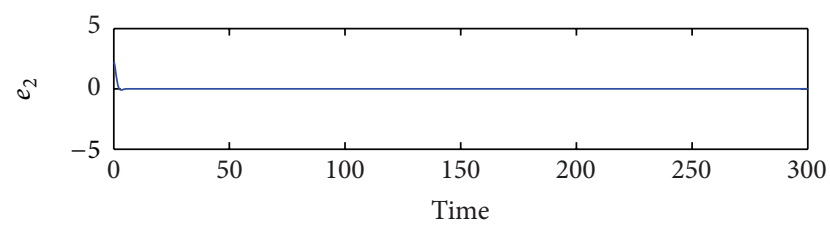

(b)

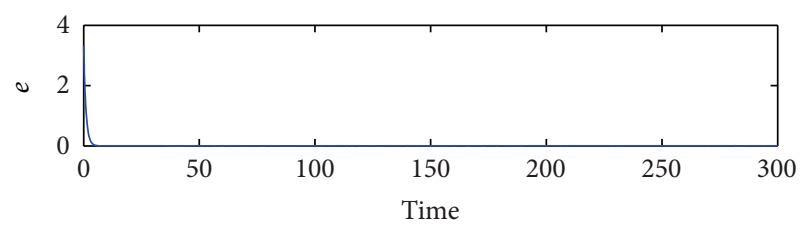

(c)

FIGURE 8: Error dynamics of the drive and the response systems with controllers activated for $t \geq 0$, where $e_{1}=w_{1}+z_{1}-\left(0.5 x_{1}+0.7 y_{1}+\right.$ $\left.0.2 x_{3}+0.3 y_{3}\right), e_{2}=w_{2}+z_{2}+\left(0.4 x_{2}+1.2 y_{2}\right)$, and $e=\sqrt{e_{1}^{2}+e_{2}^{2}}$.

master-slave synchronization involving two systems and three systems (combination synchronization) can be realized as special cases of the combination-combination synchronization scheme. The realization of a more secure communication scheme has been implemented via combination synchronization of three chaotic systems [31]. In general, the generalized reduced-order combination synchronization has potential applications in biological, social, physical, and financial systems which involve different dynamical structures and orders; hence, this type of synchronization deserves further investigation. This work confirms that the realization of combination-combination synchronization is not only limited to identical or nonidentical systems of the same order but can be realized in systems of different dynamical structures and orders.

\section{Conflict of Interests}

The authors declare that there is no conflict of interests regarding the publication of this paper.

\section{Acknowledgment}

The authors are grateful to Dr. U. E. Vincent, Department of Physics, Redeemer's University, for his valuable discussions.

\section{References}

[1] A. Singh and S. Gakkhar, "Synchronization of chaos in a food web in ecological systems," World Academy of Science, Engineering and Technology, vol. 70, pp. 94-98, 2010.
[2] G. H. Hossein, A. Asad, and K. Morteza, "Phase synchronization and synchronization frequency of two-coupled van der pol oscillators with dalay coupling," Chinese Physics B, vol. 22, no. 7, pp. 070502-070505, 2013.

[3] H.-Y. Zang, L.-Q. Min, G. Zhao, and G.-R. Chen, "Generalized chaos synchronization of bidirectionally arrays of discrete systems," Chinese Physics Letters, vol. 30, no. 4, pp. 04050210405024, 2013.

[4] C. Li and X. Liao, "Complete and lag synchronization of hyperchaotic systems using small impulses," Chaos, Solitons and Fractals, vol. 22, no. 4, pp. 857-867, 2004.

[5] W. Jawaada, M. S. M. Noorano, and M. M. Alsawalha, "Antisynchronization of chaotic systems via adaptive sliding mode control," Chinese Physics Letters, vol. 29, no. 2, pp. 120505120508, 2012.

[6] K. S. Sudheer and M. Sabir, "Hybrid synchronization of hyperchaotic Lu system," Pramana, vol. 73, no. 4, pp. 781-786, 2009.

[7] K. Z. Li, E. He, Z. R. Zeng, and K. T. Chi, "Generalized projective synchronization of coupled complex networks of different sizes," Chinese Physics B, vol. 22, no. 7, pp. 070504070511, 2013.

[8] Y. Chen and Z. Jia, "Hybrid projective dislocated synchronization of Liu chaotic system based on parameters identification," Modern Applied Science, vol. 6, no. 2, pp. 16-21, 2012.

[9] X. Wang, X. Guo, and L. Wang, "Finite-time synchronization of a new hyperchaotic lorenz system," International Journal of Modern Physics B, vol. 27, pp. 1350033-1350038, 2013.

[10] Q. -Y. Miao, J. -A. Fang, Y. Tang, and A. -H. Dong, "Increaseorder projective synchronization of chaotic systems with time delay," Chinese Physics Letters, vol. 26, no. 5, pp. 050501-050505, 2009.

[11] M. Mossa Al-sawalha and M. S. M. Noorani, "Chaos reducedorder anti-synchronization of chaotic systems with fully unknown parameters," Communications in Nonlinear Science and Numerical Simulation, vol. 17, no. 4, pp. 1908-1920, 2012.

[12] C.-C. Yang, "Robust synchronization and anti-synchronization of identical $\Phi^{6}$ oscillators via adaptive sliding mode control," Journal of Sound and Vibration, vol. 331, no. 3, pp. 501-509, 2012.

[13] F. Yu, C. Wang, Q. Wan, and Y. Hu, "Complete switched modified function projective synchronization of five-term chaotic systems with uncertain parametersand disturbances," Pramana Journal of Physics, vol. 80, no. 2, pp. 223-235, 2013.

[14] A. N. Njah, "Synchronization via active control of parametrically and externally excited $\varphi^{6}$ van der pol and duffing oscillators and application to secure communications," Journal of Sound and Vibration, vol. 17, no. 4, pp. 493-504, 2011.

[15] K. S. Ojo, A. N. Njah, and S. T. Ogunjo, "Comparison of backstepping and modified active control in projective synchronization of chaos in an extendedbonhoffer- van der pol oscillator," Pramana, vol. 80, no. 5, pp. 825-835, 2013.

[16] J. Lu, D. W. C. Ho, J. Cao, and J. Kurth, "Single impulsive controller for globally exponential synchronization of dynamical networks," Nonlinear Analysis: Real World Application, vol. 14, pp. 581-593, 2013.

[17] M. Ma, J. Zhou, and J. Cai, "Practical synchronization of nonautonomous systems with uncertain parameter mismatch via a single state feedback control," International Journal of Modern Physics C, vol. 23, no. 11, pp. 12500731-1250073114, 2012.

[18] K. S. Ojo, A. N. Njah, and S. T. Ogunjo, "Comparison of backstepping and modified active control in projective synchronization of chaos in an extended bonhoffer van der pol oscillator," Pramana Journal of Physics, vol. 80, no. 5, pp. 825-835, 2013. 
[19] A. N. Njah, K. S. Ojo, G. A. Adebayo, and A. O. Obawole, "Generalized control and synchronization of chaos in RCL-shunted Josephson junction using backstepping design," Physica C, vol. 470, no. 13-14, pp. 558-564, 2010.

[20] A. N. Njah and K. S. Ojo, "Backstepping control and synchronization of parametrically and externally excited $\Phi^{6}$ van der pol oscillators with application to secure communications," International Journal of Modern Physics B, vol. 24, no. 23, pp. 4581-4593, 2010.

[21] B. A. Idowu, U. E. Vincent, and A. N. Njah, "Generalized adaptive backstepping synchronization for non-identical parametrically excited systems," Nonlinear Analysis: Modelling and Control, vol. 14, no. 2, pp. 165-176, 2009.

[22] B. A. Idowu, U. E. Vincent, and A. N. Njah, "Synchronization of chaos in non-identical parametrically excited systems," Chaos, Solitons and Fractals, vol. 39, no. 5, pp. 2322-2331, 2009.

[23] R. Mainieri and J. Rehacek, "Projective synchronization in three-dimensional chaotic systems," Physical Review Letters, vol. 82, no. 15, pp. 3042-3045, 1999.

[24] H.-X. Wang, Q.-S. Lu, and X. Shi, "Phase synchronization and its transition in two coupled bursting neurons: theoretical and numerical analysis," Chinese Physics B, vol. 19, no. 6, pp. 060509060518, 2010.

[25] S. Wang and Y.-G. Yu, "Generalized projective synchronization of fractional order chaotic systems with different dimensions," Chinese Physics Letters, vol. 29, no. 2, pp. 020505-020508, 2012.

[26] R. Femat and J. Alvarez-Ramírez, "Synchronization of a class of strictly different chaotic oscillators," Physics Letters A, vol. 236, no. 4, pp. 307-313, 1997.

[27] D. Terman, N. Kopell, and A. Bose, "Dynamics of two mutually coupled slow inhibitory neurons," Physica D, vol. 117, no. 1-4, pp. 241-275, 1998.

[28] J. A. Laoye, U. E. Vincent, and O. O. Akigbogun, "Chaos control and reduced order synchronization of rigid body," International Journal of Nonlinear Science, vol. 6, no. 2, pp. 106-113, 2008.

[29] L. Runzi, W. Yinglan, and D. Shucheng, "Combination synchronization of three classic chaotic systems using active backstepping design," Chaos, vol. 21, no. 4, pp. 043114-043120, 2011.

[30] L. Runzi and W. Yinglan, "Active backstepping-based combination synchronization of three chaotic systems," Advanced Science, Engineering and Medicine, vol. 4, pp. 142-147, 2012.

[31] L. Runzi and W. Yinglan, "Finite-time stochastic synchronization of three different chaotic systems and its application in secure communication," Chaos, vol. 22, no. 2, pp. 023109023110, 2012.

[32] Z. Wu and X. Fu, "Combination synchronization of three different order nonlinear systems using active backstepping design," Nonlinear Dynamics, vol. 73, pp. 1863-1872, 2013.

[33] S. Wen, S. Chen, and J. Lü, "A novel hybrid synchronization of two coupled complex networks," in Proceedings of the IEEE International Symposium on Circuits and Systems (ISCAS '09), pp. 1911-1914, May 2009. 


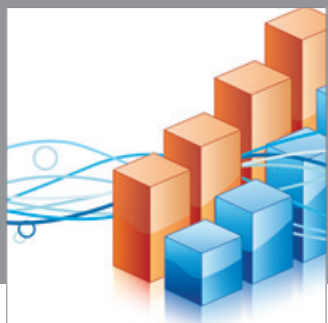

Advances in

Operations Research

mansans

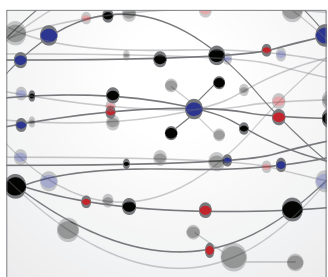

The Scientific World Journal
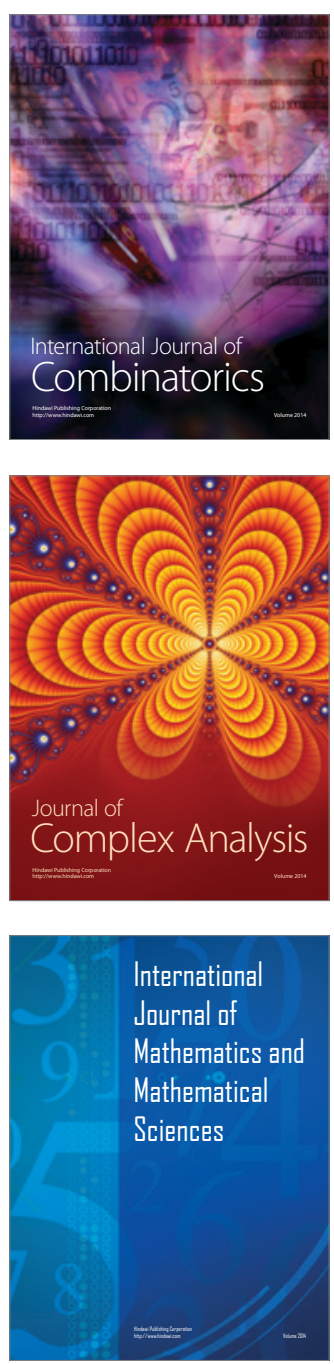
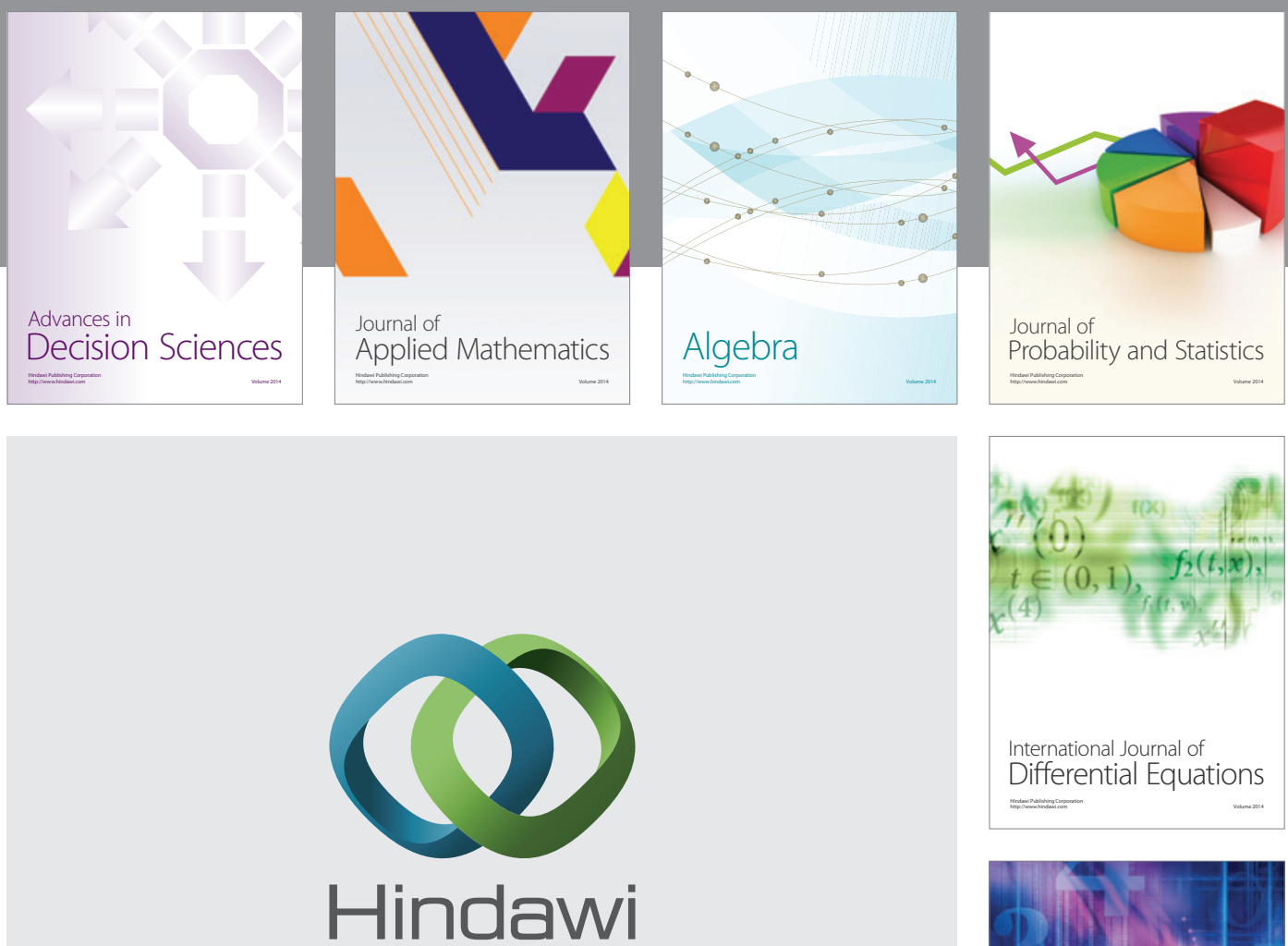

Submit your manuscripts at http://www.hindawi.com
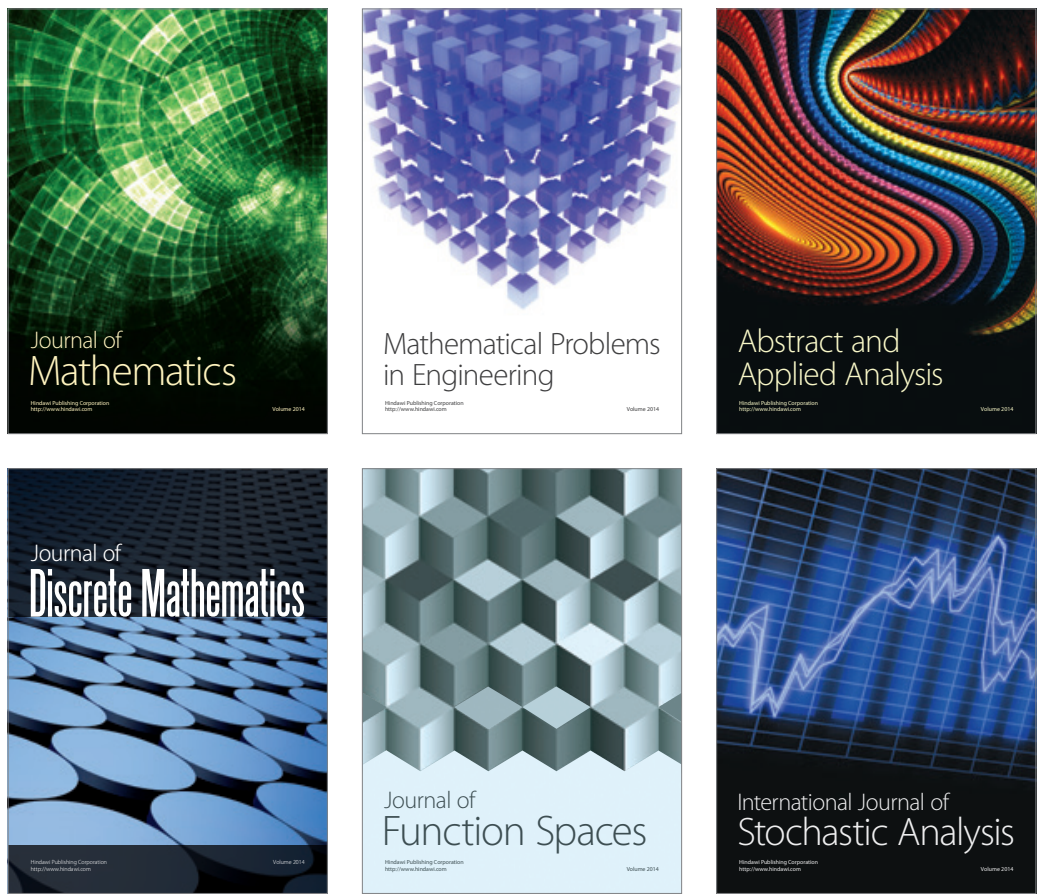

Journal of

Function Spaces

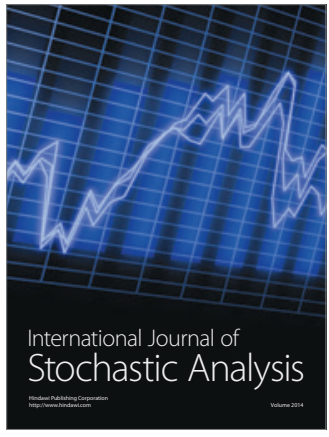

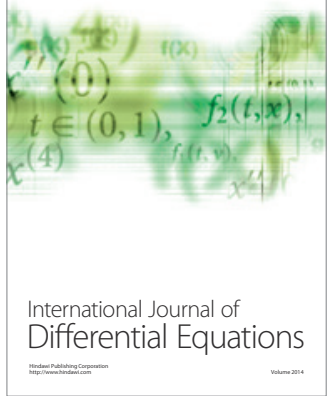
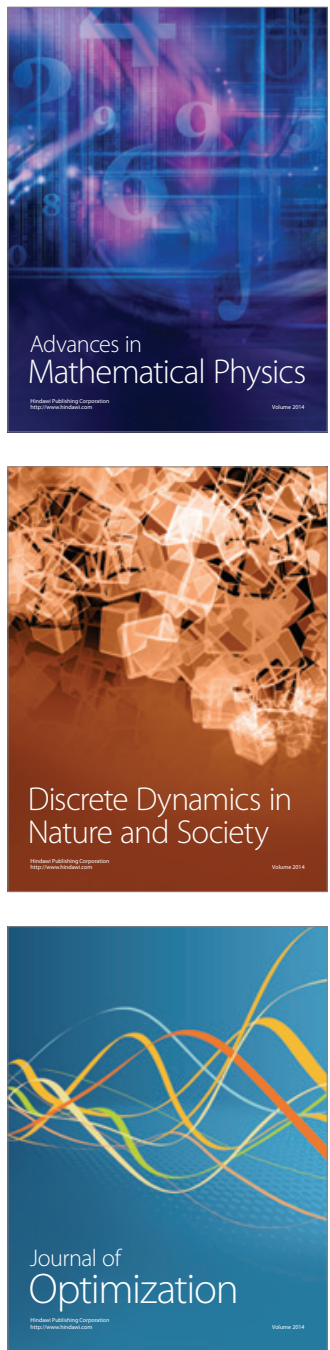